\title{
The role of sex and sex-related hormones in cognition, mood and
} well-being in older men and women

\author{
Q Q1 Teresa Costa Castanho a,b,c,1, Pedro Silva Moreira a,b,c,1, Carlos Portugal-Nunes a,b,c, \\ Ashley Novais ${ }^{a, b, c}$, Patrício Soares Costa ${ }^{a, b, c}$, Joana Almeida Palha ${ }^{a, b, c}$, Nuno Sousa ${ }^{a, b, c}$, \\ Nadine Correia Santos ${ }^{a, b, c, *}$ \\ ${ }^{a}$ Life and Health Sciences Research Institute (ICVS), School of Health Sciences, University of Minho, 4710-057 Braga, Portugal \\ b ICVS/3B's, PT Government Associate Laboratory, 4710-057 Braga/Guimarães, Portugal \\ ${ }^{\text {c } C l i n i c a l ~ A c a d e m i c ~ C e n t e r ~-~ B r a g a, ~ 4710-057 ~ B r a g a, ~ P o r t u g a l ~}$
}

\section{A R T I C L E I N F O}

\section{Article history:}

Received 23 April 2014

Accepted 27 August 2014

Available online xxx

\section{Keywords:}

Executive function

Memory

Quality of life

Sleep

Nutrition

\begin{abstract}
A B S T R A C T
Alterations in hormone levels during aging have been shown to impact on cognitive functioning and mood. However, with respect to sex and sex-related hormones research has mainly focused on testosterone and estradiol. Serum concentration levels of testosterone (TT), estradiol (E2), follicle-stimulating hormone (FSH), luteinizing hormone (LH), dehydroepiandrosterone sulfate (DHEAS) and prolactin (PRL) were assessed at fasting in 120 community-dwellers (51+ years of age, males and females), in a crosssectional approach. An extensive battery of neurocognitive/psychological and quality of life instruments was administered. Performance clusters based on executive functioning (GENEXEC), memory (MEM), mood and well-being (sleep, quality of life and nutritional status) measures were obtained. In males, higher PRL levels were associated with worse cognitive performance, lower well-being scores, and higher scores in depression scales. Additionally, E2 levels were significantly lower in individuals with higher depressive mood and poorer cognitive functioning. Linear regression analyses revealed that in males DHEAS is positively associated with GENEXEC and MEM, after adjustment of the remaining hormones and socio demographic variables. Higher PRL levels were associated with higher depressive mood and lower well-being. Nutritional status was significantly associated with PRL (positively) and with DHEAS (negatively). These findings indicate that besides the more exhaustively studied impact of E2 and TT on cognition and depression, variations in the levels of sex-related hormones such as PRL, FSH, LH and DHEAS are of interest for the mental health aging profile.
\end{abstract}

(c) 2014 Published by Elsevier B.V.

\section{Introduction}

Among older individuals, the effects of age in multiple biological systems is quite variable, with some individuals showing a more accelerated decline and others a higher "resistance" to the aging process. On this, the role of hormones remains an area of interest (Alfaro et al., 2008; Zjacic-Rotkvic, Kavur, \& Cigrovski-Berkovic, 2010), with the level and action of sex hormones, their receptors and responses, being suggested to contribute to the cognitive

\footnotetext{
* Corresponding author at: Life and Health Sciences Research Institute (ICVS),
School of Health Sciences, University of Minho, Campus Gualtar, 4710-057 Braga,

* Corresponding author at: Life and Health Sciences Research Institute (ICVS),
School of Health Sciences, University of Minho, Campus Gualtar, 4710-057 Braga, Portugal. Tel.: +351 253 604854; fax: +351253604847.

E-mail address: nsantos@ecsaude.uminho.pt (N.C. Santos).

1 These authors contributed equally in the authorship and should be listed as co-authors.
}

processing, mood and overall aging-associated processes (Alfaro et al., 2008, Boss, Kang, Marcus, \& Bergstrom, 2013; Yaffee et al., 2007). In women, changes mediated by the decline of estrogen result in increased concentrations of luteinizing hormone (LH), follicle-stimulating hormone (FSH) and prolactin (PRL). Even though not as dramatic as in females, these hormones also show increasing serum levels in males throughout aging (Feldman et al., 2002; Hermann \& Berger, 1999). Although the relations between FSH, PRL and cognition are not clear (Luetters et al., 2007), there are evidences that higher LH levels are associated with lower cognitive performance, with evidence mainly from neurodegenerative diseases, namely Alzheimer's disease (Rodrigues et al., 2008; Webber, Perry, Smith, \& Casadesus, 2007). Notably, most LH receptors in the brain are found in the hippocampus, a region particularly affected by aging and related to spatial learning and memory (Lei, Rao, Kornyei, Licht, \& Hiatt, 1993). Studies have indicated a significant loss of neurons in the hippocampus of the elderly, as well 
49 as a volumetric reduction and an altered synaptic plasticity (Prull, Gabrieli, \& Bunge, 2000). In fact, there is a well-founded association between the dimension of the hippocampus and memory performance. Memory tasks tend to present less hippocampal activation in older adults than in young subjects (Prull et al., 2000). Furthermore, FSH is also of interest, with high endogenous levels of FSH being noted in older women with well-preserved cognitive functioning (Rodrigues et al., 2008).

Like estrogen, testosterone (TT), also found to progressively decline with age, is considered a "brain-boosting" hormone influencing mood, memory and overall cognition (Aleman et al., 2001; Boss et al., 2013). Higher levels of TT have been associated with better verbal memory and visual memory and function (Boss et al., 2013), but with decreased performance on fluid intelligence (Aleman et al., 2001). In older women, higher levels of TT predicted better performance on several cognitive tests (BarrettConnor \& Goodman-Gruen, 1999). In the body, this hormone tends to bind with sex-hormone binding globulin (SHBG) and, to a lesser extent, weakly to non-specific proteins such as albumin, but some testosterone remains freely circulating in the bloodstream (Moffat et al., 2002). Higher bioavailable (free and albumin-bound) and free testosterone concentrations have each been related with better performance in distinct features of memory and cognitive functioning (Yeap et al., 2008). Testosterone exerts its neuromodulatory influence through its metabolization into E2 or dihydrotestosterone (DHT) that can bind to androgen receptors in specific brain regions, such as the hippocampus, amygdala and prefrontal cortex 76Q3 (Janowsky, 2006).

Of further relevance, dehydroepiandrosterone (DHEA), a potent neurosteroid, and its sulfated metabolite (DHEAS), precursors in estrogen and testosterone synthesis, continuously and gradually decline with advancing age (Maggio et al., 2014; Zjacic-Rotkvic et al., 2010). Studies on these are very scarce, but DHEA has been proposed to have an anti-aging effect and to represent a biomarker of healthy aging (Rathna \& Padma, 2013). It was recently shown that its increased levels might be associated with lower depressive symptoms and better performance in memory and overall cognitive ability in older men and women (Goldstein, Holsen, Handa, \& Tobet, 2013). More specifically, higher concentrations seem related with a better performance in executive function, concentration and working memory, in post-menopausal women (Davis, Panjari, \& Stanczyk, 2011). Finally, the decline in DHEAS appears to have an influence on the quality of life, not only in the physical functioning and mental health but also in social aspects (Martínez-Jabaloyas et al., 2011) and in mood (Harsh, Meltzer-Brody, Rubinow, \& Schmidt, 2009). Its potential involvement in depression is relevant when considering the more pronounced decrease in the levels of DHEA that occur in aging women when compared to males (Harsh et al., 2009), and that older females overall present a more depressed mood (Santos et al., 2013).

Herein, we aimed to assess the relation between TT, estradiol (E2), FSH, LH, PRL and DHEAS levels with cognitive functioning, mood status and overall quality of life in a cohort of healthy aging individuals.

\section{Materials and methods}

\subsection{Subjects}

Participants $(n=120)$ were randomly selected from the Guimarães and Vizela local area health authority registries in the North of Portugal, and were representative (gender and age) of an original cohort representative of the health registries and of the general Portuguese population (Costa, Santos, Cunha, Palha, \& Sousa, 2013; Santos et al., 2013, 2014). This database differs in less than $2 \%$ of that of the distribution for the Portuguese population estimated by the Portuguese authority on statistics, the Instituto Nacional de Estatística (INE). The study was conducted in accordance with the Declaration of Helsinki and approved by national and local ethics review boards. Potential participants were contacted by telephone to participate in the study, by the researchers. The goals and nature of the evaluation were explained to all potential participants. Of the 246 individuals contacted, 120 that met inclusion/exclusion criteria accepted to participate in the study. All participants provided free informed consent. The primary exclusion criteria included inability to understand informed consent, participant's choice to withdraw from the study, incapacity and/or inability to attend the neuropsychological assessment session(s), diagnosed dementia or neuropsychiatric disorder. Clinical measures were self-reported and confirmed from medical records. Assessments were conducted at the Clinical Academic Center - Braga.

\subsection{Hormone measurements}

Blood samples were collected in the morning (between 8:30 and 9:30 am) at the Hospital de Braga, after an overnight fast. Serum concentration of testosterone (tota testosterone, bound and unbound, TT, ng/dl, ADVIA Centaur, Siemens), estradio (E2, pmol/l, ADVIA Centaur, Siemens), follicle-stimulating hormone (FSH, $\mathrm{mIU} / \mathrm{ml}$ ADVIA Centaur, Siemens, Frimley, Camberly, UK), luteinizing hormone ( $\mathrm{LH}, \mathrm{mIU} / \mathrm{ml}$; ADVIA Centaur, Siemens), prolactin (PRL, mIU/ml; ADVIA Centaur, Siemens) and dehydroepiandrosterone sulfate (DHEA-SO4, $3 \mu \mathrm{g} / \mathrm{dl}$; Immulite 2000, Siemens) were determined by chemiluminescent immunoassays using commercial kits and following the manufacturers' instructions. For these hormones the intra-assay coefficients of variation and detection limits were, respectively: intra-assay coefficients of variation $7 \%, 12 \%,<3 \%,<4 \%,<5 \%$ and $<10 \%$; detection limits $10 \mathrm{ng} / \mathrm{dl}, 43.6 \mathrm{pmol} / \mathrm{l}$ $0.3 \mathrm{mIU} / \mathrm{ml}, 0.07 \mathrm{mIU} / \mathrm{ml}, 0.07,6.4 \mathrm{IU} / \mathrm{ml}$ and $3 \mu \mathrm{g} / \mathrm{dl}$; undetectable limits in the total sample $1.7 \%, 2.5 \%, 2.5 \%, 1.7 \%, 2.5 \%$ and $1.7 \%$.

\subsection{Neurocognitive/psychological assessment}

A team of trained psychologists conducted the neurocognitive/psychologica assessments. Tests were selected to provide mood and cognitive [general cognitive status and executive (GENEXEC) and memory (MEM) functions] profiles, as previously reported (Costa et al., 2013; Paulo et al., 2011; Santos et al., 2013). Briefly, the following cognitive measures were used: global cognitive status was assessed with the mini-mental state examination (MMSE); short-term verbal memory with the digit span forward test (Digits Fw; subtest of the Wechsler adult intelligence test WAIS III), working memory with the digit span backward test (Digits Bw; subtest of the Wechsler adult intelligence test WAIS III), multiple trial verbal learning and memory with the selective reminding test [SRT, parameters: consistent long term retrieval (CLTR), long term storage (LTS), delayed recall (DR) and intrusions] and the Consortium to Establish a Registry for Alzheimer's Disease-Word List test [CERAD, parameters: Total hits, Total intrusions, DR hits, DR intrusions, Recognition (REC) hits, Recognition (REC) rejections]; verbal fluency with the controlled ora word association test F-A-S [COWAT-FAS, parameters: admissible (Ad) and nonadmissible (NAd)]; response inhibition/cognitive flexibility with the Stroop color and word test (parameters: Words, Colors and Words/colors); and, high-level information processing speed with the digit symbol substitution test (DSST, subtest of the Wechsler adult intelligence test WAIS III, 1997). The geriatric depression scale (GDS, long-version) was used for evaluation of depressive mood. All instruments were administered in Portuguese (the primary and native language of the participants).

To assess quality of life the following measures were used: the WHO Quality of Life-BREF (WHOQOL-BREF) (Vaz Serra et al., 2006) for overall quality of life domains (physical and psychological health, social relationships and environment; as well as global quality of life and general health); the Pittsburgh Sleep Quality (PSQI) (Buysse, Reynolds, Monk, Berman, \& Kupfe, 1988) for sleep quality and disturbances on various domains (subjective sleep quality, sleep latency, sleep duration, habitual sleep efficiency, sleep disturbances, use of sleeping medication and daytime dysfunction); and, the Full Mini-Nutritional Assessment (F-MNA) (Guigoz, Vellas, \& Garry, 1996) to identify (overt) malnutrition or the risk of malnutrition. For the WHOQOL and the F-MNA questionnaires, the higher the score the better the status of the measured parameter; for the PSQI, higher scores represent worse sleep quality.

\subsection{Data analyses}

All variables (neurocognitive, neuropsychological and quality of life measures) were expressed in the same scale by conversion into $z$ scores. Independent samples $t$-test were performed to analyze the differences in hormone levels, sociodemographic variables, cognitive functioning, mood and quality of life measures between men and women. Principal component analysis (PCA) was conducted to reduce the number of variables with a minimum loss of information and, therefore, reduce the number of comparisons. The variables included in PCA analysis are described in the results section. The number of retained components was determined by Kaiser criterion (eigenvalue $>1$ ). Factor scores were obtained (using the regression method) and were used in subsequent analysis. The reliability of each component was analyzed using Cronbach's alpha. Hierarchical cluster analysis (Ward's method based on squared Euclidean distances) was conducted to identify profiles based on PCA's factor scores and on the GDS total score. For this purpose analyses of variance were conducted in order to compare each cluster with respect to the mentioned measures within different solutions. The remaining statistical analysis was conducted separately for males and females. A multinomial logistic regression was conducted in order to test if hormone levels could predict cluster 
membership. Multiple linear regressions controlling for age and education were performed between hormone levels and cognitive, mood and well-being measures. Statistical significance was defined at $p<.05$ level. Statistical analysis was conducted using the SPSS package v21 (IBM SPSS Statistics).

\section{Results}

\subsection{Sample characteristics}

The mean age of the population was 65.2 years (range, 51-87 years; $\mathrm{SD}=8.8$; age categories: [50-60[, 30\% (males, 63.9\%); [60-70[, 35.8\% (males, 46.5\%); [70+[, 34.2\% (males, 48.8\%)), with $n=63(52.5 \%)$ males. The median years of schooling was 4; specifically, 23.3\% (males 25.0\%), 53.3\% (males 54.7\%), and $23.3 \%$ (males $75 \%$ ) of the cohort had [0-4[, 4, and [4+[years of school education (elementary school), respectively. All participants lived in the community (community-dwellers), with equal distribution among rural and urban areas. On socio-demographic measures, Portugal ranks close to the OECD (Organization for Economic Co-operation and Development; www.oecd.org/) average (OECD, 2012). Descriptive statistics for hormone levels, scores on neurocognitive/psychological tests, mood (depression), and overall quality of life, sleep quality and nutritional status are presented in Table 1. All females were post-menopausal. Participants were not currently using any hormone therapy. The values are shown for the overall sample and separately for males and females. The significance of each difference between genders is presented.

From the PCA, two cognitive dimensions were obtained. The "MEM" dimension (memory function, Cronbach's alpha: 0.917), composed of the SRT (CLTR and LTS) and the CERAD (total hits and delayed recall) parameters, and the "GENEXEC" dimension (general executive function, Cronbach's alpha: 0.873) composed of the MMSE (total score), Stroop (words, color and combination) and digits span (direct and backward orders) parameters. For "MEM", the parameter intrusions, in both the SRT and the CERAD tests, were excluded from the model due to low communalities. Delayed recall in the SRT was also excluded due to the presence of missing values. For "GENEXEC", the score of total admissible words in the COWAT had low communalities in the solution and was excluded. A "WELL-BEING" dimension (Cronbach's alpha: 0.792) was obtained composed of the dimensions of the WHOQOL (Physical, Psychological, Social and Environment), PSQI and the F-MNA (total scores) parameters.

\subsection{Clusters of performance}

Based on the changes observed in the agglomeration schedule (i.e. displays the clusters combined at each stage, the distances between the clusters being combined, and the last cluster level at which an individual joined the cluster), solutions of 2, 3 and 4 clusters were retained. The 2-clusters solution divided homogenously the sample between both clusters. However, it was considered to be poorly discriminative of the measures. The 3-clusters solution was more discriminative but the distribution of the individuals on each cluster was heterogeneous. The 4-clusters solution was considered to best discriminate between measures and to produce a satisfactory division of individuals between clusters and was, therefore, selected for the subsequent analysis. This solution followed previous findings regarding patterns of cognitive performance for larger cohorts with similar characteristics (Costa et al., 2013; Santos et al., 2013), where mood could further subdivide cognitive performance. The cohort replicated the findings (Fig. 1).

Specifically, the first cluster (C1) was characterized by high cognition (in executive function and memory dimensions), high well-being and low depressive mood; the second cluster (C2)

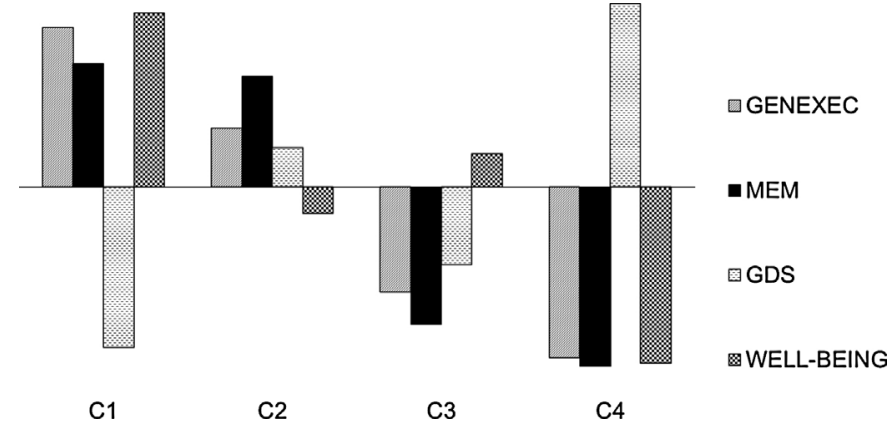

Fig. 1. Mean performance $z$-scores by clusters in the GENEXEC, MEM, GDS and WELL-BEING dimensions. GENEXEC: general executive function; MEM: memory function; GDS: Geriatric Depression Scale.

encompassed moderate-high levels of cognition, moderate-high well-being and moderate-high depressive mood; the third cluster (C3) was composed of individuals with moderate-low levels of cognition, moderate-high levels of well-being and moderatelow levels of depressive mood; and, the fourth cluster (C4) was characterized by low cognition, low overall well-being and high depressive mood. As observed in the ANOVA (Table 2), "GENEXEC", mood and well-being were significantly different between all clusters. For "MEM", no significant differences were observed between $\mathrm{C} 1$ and $\mathrm{C} 2$ and between $\mathrm{C} 3$ and $\mathrm{C} 4$, with the remaining comparisons being significant.

\subsection{Sex hormones and cluster performance}

The multinomial logistic regression (Table 3 ) revealed that in males, adjusting for age and education, E2 predicted significantly cluster membership between $\mathrm{C} 4$ and both $\mathrm{C} 1$ ( OR $=1.11, p=.039$ ) and C3 $(\mathrm{OR}=1.11, p=.025)$, i.e. participants with higher estradiol levels are more likely to be on $\mathrm{C} 1$ and $\mathrm{C} 3$, comparing to C4. There was also a trend for individuals with higher E2 levels to present a higher chance to be on $\mathrm{C} 2$ when compared to $\mathrm{C} 4$ $(\mathrm{OR}=1.10, p=.053)$. Higher PRL levels present a significantly higher chance for participants to be on C4 comparing to $\mathrm{C} 1$ ( $\mathrm{OR}=0.56$, $p=.014)$, and individuals with higher TT levels present a decreased chance of being on $\mathrm{C} 4$ comparing to $\mathrm{C} 2(\mathrm{OR}=0.98, p=.034)$ and C3 (OR=0.98, $p=.043)$. In females, higher LH levels were significantly associated with a lower chance of being on C3 when compared to $\mathrm{C} 4(\mathrm{OR}=0.76, p=.039)$. A trend was also noted for FSH to significantly predict membership between these clusters $(\mathrm{OR}=1.12, p=.056)$, with higher FSH levels being associated with an increased chance of being on $\mathrm{C} 3$ comparing to $\mathrm{C} 4$; however, these results lose statistical significance after adjustment for age and education.

\subsection{Sex hormones and cognitive, mood and quality of life measures}

Linear regression analyses indicated that in males, sex hormones, age and education (set as independent variables), significantly predict the different domains assessed, with the exception of PSQI. Specifically, education and DHEAS were positive significant predictors of GENEXEC and MEM; education (positive) and PRL (negative) were significant predictors of GDS and WELLBEING; education was a positive significant predictor of WHOQOL; education (positive), PRL (negative) and DHEAS (positive) were significant predictors of F-MNA. In females, for the same predictors and dimensions, education was observed as a positive significant predictor of GENEXEC and MEM dimensions specifically, with no significant findings for any other dimension (Table 4). Partial 
Table 1

Descriptive statistics.

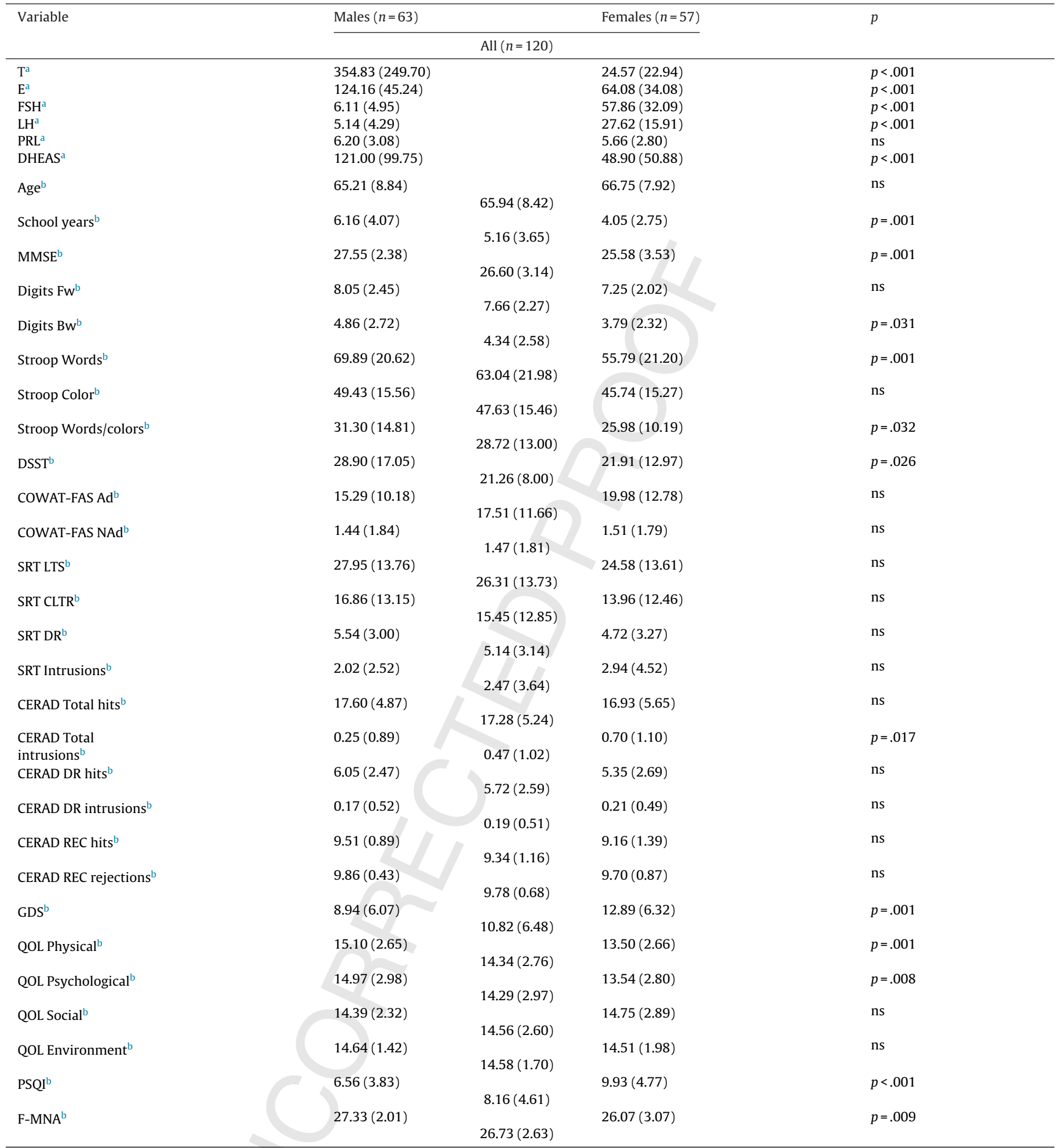

a Values presented in median (IQR).

b Values presented in mean (SD).

All the differences in cognitive performance between genders are no longer significant after controlling for education.

correlations between hormone levels and cognitive, mood and well-being measures, controlling for age and education, are presented in Supplementary Table S1. Complementing the findings, in males FSH and LH were significantly associated with executive functioning ( $\mathrm{FSH}, r=-.311, p=.018$; $\mathrm{LH}, r=-.319, p=.015)$, while in respect to females, there was a trend for a significant relationship between LH and PSQI $(r=.275, p=.056)$.

Supplementary Table S1 related to this article can be found, in the online version, at http://dx.doi.org/10.1016/ j.biopsycho.2014.08.015. 
Table 2

Means of GENEXEC, MEM, GDS and WELL-BEING dimensions for each cluster.

\begin{tabular}{|c|c|c|c|c|}
\hline & GENEXEC $^{\mathrm{a}}$ & MEM $^{a}$ & $\mathrm{GDS}^{\mathrm{a}}$ & WELL-BEING \\
\hline C1 $(n=29)$ & $1.00(0.52)$ & $0.77(0.49)$ & $-1.00(0.37)$ & $1.09(0.45)$ \\
\hline$C 2(n=40)$ & $0.37(0.83)$ & $0.69(0.64)$ & $0.24(0.76)$ & $-0.17(0.56)$ \\
\hline C3 $(n=24)$ & $-0.66(0.46)$ & $-0.86(0.46)$ & $-0.49(0.49)$ & $0.21(0.68)$ \\
\hline$C 4(n=26)$ & $-1.07(0.44)$ & $-1.12(0.42)$ & $1.14(0.75)$ & $-1.10(0.92)$ \\
\hline$F$ & 65.19 & 103.58 & 52.59 & 59.43 \\
\hline$p$ & $<.001$ & $<.001$ & $<.001$ & $<.001$ \\
\hline
\end{tabular}

a Values presented in mean (SD).

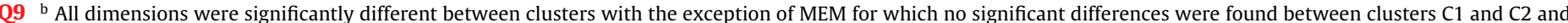
clusters $\mathrm{C} 3$ and $\mathrm{C} 4$.

GENEXEC: $\mathrm{C} 1>\mathrm{C} 2>\mathrm{C} 3>$ C4; MEM: $\mathrm{C} 1 / \mathrm{C} 2>\mathrm{C} 3 / \mathrm{C} 4$; GDS: $\mathrm{C} 1<\mathrm{C} 3<\mathrm{C} 2<\mathrm{C} 4$; WELL-BEING: $\mathrm{C} 1>\mathrm{C} 3>\mathrm{C} 2>\mathrm{C} 4$.

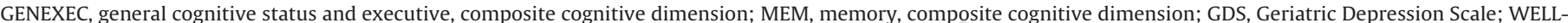
BEING, well-being, composite quality of life, quality of sleep, and nutritional status dimension.

\section{Discussion}

In a community-dwellers sample, we investigated, in both men and women, the association between serum concentration levels of sex and sex-related hormones with variables encompassing cognitive, psychological and well-being domains (the latter including overall quality of life, sleep and nutritional status variables). For this purpose, and to maximize the statistical power of the model,

Table 3

Logistic regression analysis with the binary/multinomial dependent variable cluster membership. ${ }^{\text {a }}$

\begin{tabular}{|c|c|c|c|c|c|c|c|c|}
\hline & \multicolumn{4}{|l|}{ Unadjusted } & \multicolumn{4}{|c|}{ Adjusted for age and school years } \\
\hline & \multirow[t]{2}{*}{ Odd's ratio } & \multicolumn{2}{|l|}{$95 \% \mathrm{CI}$} & \multirow[t]{2}{*}{$p$} & \multirow[t]{2}{*}{ Odd's ratio } & \multicolumn{2}{|l|}{$95 \% \mathrm{CI}$} & \multirow[t]{2}{*}{$p$} \\
\hline & & Lower & Upper & & & Lower & Upper & \\
\hline \multicolumn{9}{|l|}{ Males } \\
\hline \multicolumn{9}{|l|}{$\mathrm{C} 1$} \\
\hline $\mathrm{T}$ & 0.99 & 0.98 & 1.00 & .064 & 0.98 & 0.97 & 1.00 & .064 \\
\hline $\mathrm{E}$ & 1.08 & 1.01 & 1.16 & .028 & 1.11 & 1.01 & 1.22 & .039 \\
\hline FSH & 0.99 & 0.77 & 1.26 & .909 & 0.80 & 0.52 & 1.23 & .298 \\
\hline LH & 0.63 & 0.35 & 1.11 & .110 & 0.77 & 0.26 & 2.29 & .638 \\
\hline PRL & 0.69 & 0.48 & 0.99 & .042 & 0.56 & 0.36 & 0.89 & .014 \\
\hline DHEAS & 1.01 & 0.99 & 1.03 & .387 & 1.01 & 0.97 & 1.04 & .781 \\
\hline \multicolumn{9}{|l|}{$\mathrm{C} 2$} \\
\hline $\mathrm{T}$ & 0.99 & 0.97 & 1.00 & .030 & 0.98 & 0.97 & 1.00 & .034 \\
\hline $\mathrm{E}$ & 1.07 & 1.00 & 1.15 & .055 & 1.10 & 1.00 & 1.20 & .053 \\
\hline FSH & 0.92 & 0.72 & 1.17 & .499 & 0.84 & 0.58 & 1.23 & .371 \\
\hline $\mathrm{LH}$ & 0.69 & 0.41 & 1.16 & .163 & 0.73 & 0.26 & 2.08 & .554 \\
\hline PRL & 0.79 & 0.61 & 1.03 & .077 & 0.73 & 0.51 & 1.04 & .084 \\
\hline DHEAS & 1.01 & 0.99 & 1.02 & .595 & 1.00 & 0.97 & 1.03 & .918 \\
\hline \multicolumn{9}{|l|}{$\mathrm{C} 3$} \\
\hline $\mathrm{T}$ & 0.99 & 0.97 & 1.00 & .026 & 0.98 & 0.97 & 1.00 & .043 \\
\hline E & 1.09 & 1.01 & 1.17 & .020 & 1.11 & 1.01 & 1.22 & .025 \\
\hline FSH & 1.07 & 0.86 & 1.33 & .541 & 1.03 & 0.74 & 1.45 & .846 \\
\hline $\mathrm{LH}$ & 0.60 & 0.34 & 1.08 & .087 & 0.56 & 0.20 & 1.58 & .272 \\
\hline PRL & 0.80 & 0.62 & 1.04 & .096 & 0.83 & 0.64 & 1.07 & .150 \\
\hline DHEAS & 1.00 & 0.98 & 1.02 & .744 & 1.00 & 0.97 & 1.03 & .850 \\
\hline \multicolumn{9}{|l|}{ Females } \\
\hline \multicolumn{9}{|l|}{ C1 } \\
\hline $\mathrm{T}$ & 0.99 & 0.95 & 1.04 & .811 & 1.00 & 0.93 & 1.06 & .895 \\
\hline E & 0.99 & 0.96 & 1.03 & .758 & 0.99 & 0.93 & 1.05 & .643 \\
\hline FSH & 0.97 & 0.89 & 1.05 & .431 & 0.99 & 0.89 & 1.10 & .823 \\
\hline $\mathrm{LH}$ & 1.06 & 0.92 & 1.23 & .427 & 1.02 & 0.82 & 1.25 & .880 \\
\hline PRL & 1.07 & 0.75 & 1.52 & .723 & 1.12 & 0.67 & 1.87 & .667 \\
\hline DHEAS & 1.03 & 1.00 & 1.06 & .064 & 1.03 & 0.99 & 1.07 & .165 \\
\hline \multicolumn{9}{|l|}{$\mathrm{C} 2$} \\
\hline $\mathrm{T}$ & 1.00 & 0.96 & 1.04 & .870 & 0.97 & 0.93 & 1.02 & .257 \\
\hline E & 1.01 & 0.98 & 1.03 & .627 & 1.01 & 0.99 & 1.03 & .462 \\
\hline FSH & 0.99 & 0.93 & 1.06 & .789 & 0.97 & 0.91 & 1.04 & .366 \\
\hline LH & 1.04 & 0.93 & 1.18 & .475 & 1.13 & 0.97 & 1.31 & .124 \\
\hline PRL & 0.89 & 0.66 & 1.19 & .422 & 0.86 & 0.62 & 1.19 & .356 \\
\hline DHEAS & 1.01 & 0.99 & 1.04 & .293 & 1.02 & 0.99 & 1.06 & .122 \\
\hline \multicolumn{9}{|l|}{$\mathrm{C} 3$} \\
\hline $\mathrm{T}$ & 1.02 & 0.95 & 1.09 & .543 & 1.04 & 0.94 & 1.15 & .412 \\
\hline E & 1.01 & 0.98 & 1.05 & .429 & 1.02 & 0.98 & 1.06 & .309 \\
\hline FSH & 1.12 & 1.00 & 1.26 & .056 & 1.21 & 0.97 & 1.50 & .086 \\
\hline $\mathrm{LH}$ & 0.76 & 0.58 & 0.99 & .039 & 0.65 & 0.40 & 1.04 & .072 \\
\hline PRL & 0.94 & 0.54 & 1.64 & .822 & 0.97 & 0.51 & 1.83 & .921 \\
\hline DHEAS & 0.98 & 0.94 & 1.03 & .473 & 0.97 & 0.90 & 1.03 & .304 \\
\hline
\end{tabular}

a The multinomial logistic regression was performed using $\mathrm{C}^{\prime}$ ' as the reference category.

${ }^{\mathrm{b}}$ Adjusted for age and school years. 
Table 4

Multiple linear regression analysis.

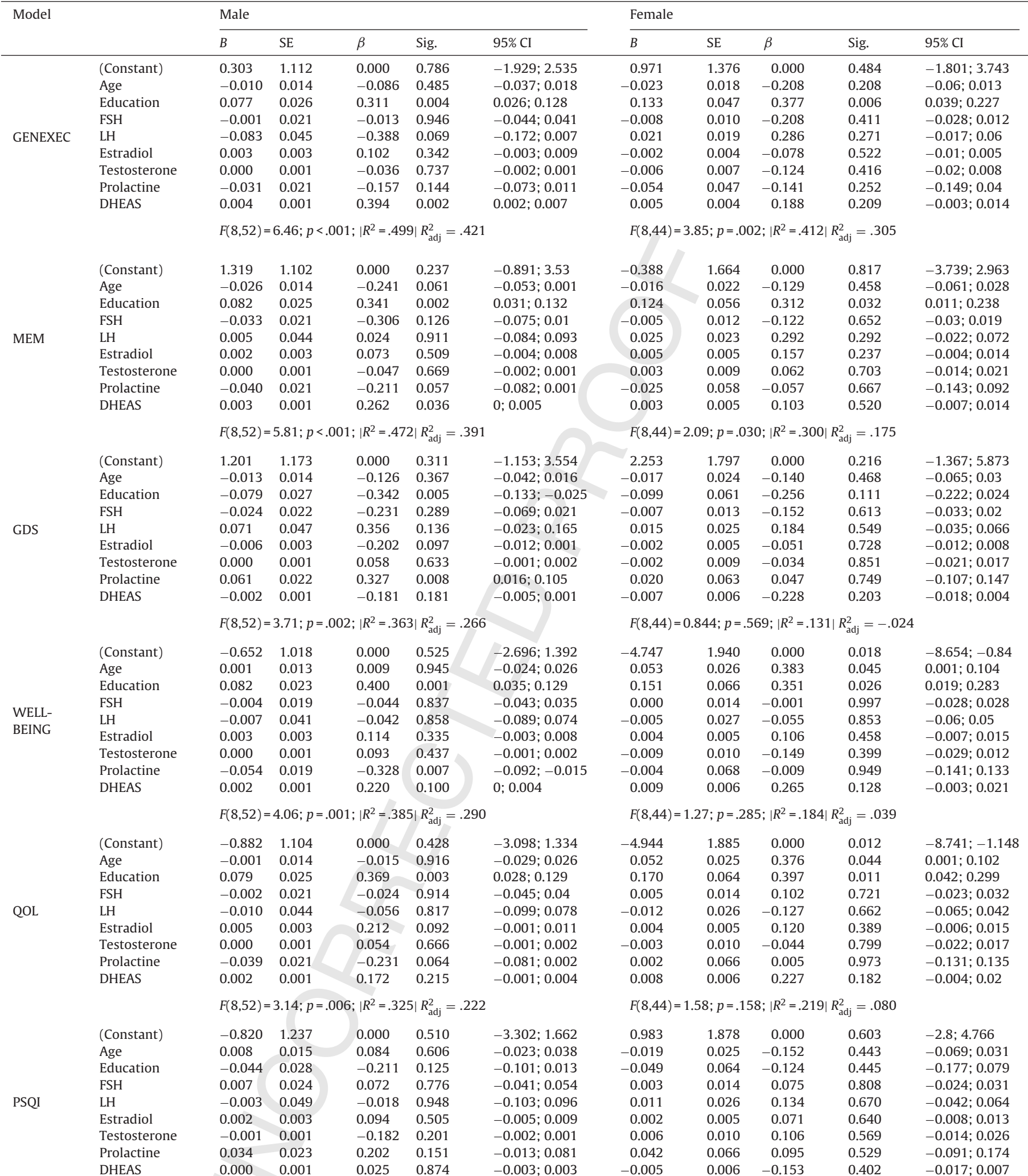


Table 4 (Continued)

\begin{tabular}{|c|c|c|c|c|c|c|c|c|c|c|c|}
\hline \multirow[t]{2}{*}{ Model } & & \multicolumn{5}{|l|}{ Male } & \multicolumn{5}{|c|}{ Female } \\
\hline & & $B$ & SE & $\beta$ & Sig. & $95 \% \mathrm{CI}$ & $B$ & SE & $\beta$ & Sig. & $95 \% \mathrm{CI}$ \\
\hline \multirow{10}{*}{ F-MNA } & (Constant) & -1.062 & 0.945 & 0.000 & 0.266 & $-2.958 ; 0.834$ & -3.558 & 2.140 & 0.000 & 0.103 & $-7.867 ; 0.751$ \\
\hline & Age & 0.018 & 0.012 & 0.204 & 0.136 & $-0.006 ; 0.041$ & 0.043 & 0.028 & 0.293 & 0.135 & $-0.014 ; 0.1$ \\
\hline & Education & 0.053 & 0.022 & 0.277 & 0.017 & $0.01 ; 0.097$ & 0.058 & 0.072 & 0.126 & 0.430 & $-0.088 ; 0.204$ \\
\hline & FSH & -0.005 & 0.018 & -0.055 & 0.793 & $-0.041 ; 0.031$ & -0.013 & 0.015 & -0.244 & 0.420 & $-0.044 ; 0.019$ \\
\hline & $\mathrm{LH}$ & -0.008 & 0.038 & -0.046 & 0.842 & $-0.083 ; 0.068$ & 0.026 & 0.030 & 0.271 & 0.382 & $-0.034 ; 0.087$ \\
\hline & Estradiol & -0.002 & 0.003 & -0.101 & 0.391 & $-0.008 ; 0.003$ & 0.005 & 0.006 & 0.115 & 0.438 & $-0.007 ; 0.016$ \\
\hline & Testosterone & 0.000 & 0.001 & 0.078 & 0.508 & $-0.001 ; 0.001$ & -0.023 & 0.011 & -0.374 & 0.045 & $-0.046 ;-0.001$ \\
\hline & Prolactine & -0.058 & 0.018 & -0.379 & 0.002 & $-0.094 ;-0.023$ & 0.015 & 0.075 & 0.028 & 0.847 & $-0.136 ; 0.166$ \\
\hline & DHEAS & 0.004 & 0.001 & 0.425 & 0.002 & $0.001 ; 0.006$ & 0.009 & 0.007 & 0.254 & 0.159 & $-0.004 ; 0.023$ \\
\hline & & \multicolumn{5}{|c|}{$F(8,52)=4.26 ; p=.001 ;\left|R^{2}=.396\right| R_{\mathrm{adj}}^{2}=.303$} & \multicolumn{5}{|c|}{$F(8,44)=.776 ; p=.626 ;\left|R^{2}=.121\right| R_{\mathrm{adj}}^{2}=-.035$} \\
\hline
\end{tabular}

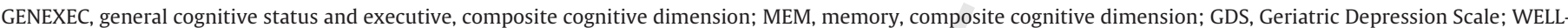
BEING, well-being, composite quality of life, quality of sleep, and nutritional status dimension.

the analysis was focused on the role of TT, E2, FSH, LH, DHEAS and PRL in the above-referred composite dimensions. Specifically, in the linear regression analysis PRL and DHEAS were the most significant predictors of the different dimensions assessed.

Overall, from all findings, PRL was found to have the strongest associations with the explored variables. Particularly in men, higher PRL serum concentrations were associated with poorer cognition, well-being and mood. PRL is well described in the literature for its role in the regulation of parental behavior, mainly with respect to the production of milk proteins, lactose and lipids, in females. It is also known for its involvement in the maintenance of cellular morphology, increasing the number of LH and FSH receptors, and in spermatogenesis (Bole-Feysot, Goffin, Edery, Binart, \& Kelly, 1998). However, the relationship between PRL and cognition, mood or quality of life has received little attention overall, and particularly in aging studies. Here we show its negative association with memory, executive function and multiple well-being domains, which agrees with other lines of evidence. It was recently shown that the secretion of PRL, along with corticotrophin releasing hormone, glucocorticoids or oxytocin, reduces neurogenesis and modulates neuroendocrine function in the adult (Lajud et al., 2013). A possible explanation regarding the significant association between PRL and cognition may stem from the modulatory role that dopamine (DA) exerts on PRL (Ben-Jonathan \& Hnasko, 2001). DA is critically implicated in many cognitive abilities, as well as age-related cognitive deficits. Furthermore, neuroimaging studies have revealed strong relationships between DA receptors (both for $D_{1}$ and $D_{2}$ receptors) and performance in several psychomotor tests (Wang et al., 1998; Yang et al., 2003). Still, in human studies, to our knowledge, there is only one report that examines the association between PRL and cognitive functioning. Henry and Sherwin (2012) report that higher PRL levels had a detrimental effect in executive functioning, as we also observe here. In accordance, in animal studies, reduced PRL levels in mice seem to compromise learning and memory behaviors that require input from the hippocampus (Walker et al., 2012), and in male rats hyperprolactinemia impairs object recognition (Torner, Tinajero, Lajud, Quintanar-Stephano, \& Olvera-Cortes (2013)). The reason why we could only verify these relationships in males (across all dimensions) remains to be explored, particularly as an association between hyperprolactinemia and increased levels of depression, anxiety and decreased libido has been reported in women (Lisansky et al., 1984). Finally, it has also been shown that dietary improvement lowers the serum concentration levels of PRL (Lunn, Austin, Prentice, \& Whitehea, 1984), which is in agreement with the inverse relationship of nutritional status and PRL levels found in the present study.

Following the PRL results, DHEAS was also associated with multiple domains, particularly on measures of executive functioning and of well-being, in males, with higher levels of DHEAS being positively associated with the mentioned dimensions. These findings are consistent with reports on the positive association between DHEAS concentration and visuospatial abilities, verbal fluency and short-term memory (Rathna \& Padma, 2013), suggesting a favorable role in cognitive aging. In animal models, DHEAS administration increased acetylcholine levels in the hippocampus of male rats, being hypothesized that it might promote neuroprotective mechanisms (Farr, Banks, Uezu, Gaski, \& Morley, 2004). Further, no significant relationships were here found between DHEAS and mood. This is an unexpected result since, from the literature, DHEAS is among all hormones here addressed the one that presents the most consistent results concerning depressive mood (Wong et al., 2011). This association can be interpreted based on an increase in neuronal "excitability", producing effects on neurotransmitter systems similar to those of anti-depressive medication (Morsink et al., 2007). It has also been suggested that DHEAS may impact on immunological and parameters, through an anti-glucocorticoid action. Specifically, DHEAS is reported to stimulate the immune system, inhibit the inflammatory process, enhance neuronal plasticity and display neuroprotective properties (Wolf \& Kirschbaum, 1999). Thus, low levels of DHEAS may make older individuals more susceptible to the recognized detrimental effects of high levels of cortisol. Finally, other studies have suggested that DHEAS positively influences endocrine and metabolic mechanisms (Ravaglia et al., 1996), which may explain the relationship between DHEAS and better nutritional status observed in our study.

Although TT was not significantly associated with any of the studied dimensions individually, it differentiated cluster membership in males, after adjustment for age and education. Interestingly, we found opposite associations between TT levels and mood status for "good" (C1 and C2) and "poor" (C3 and C4) cognitive performers. Specifically, among good cognitive performers, individuals with lower depressive mood presented higher serum TT concentration, whereas for poor performers, those with more depressive mood presented higher TT serum levels. Results from other studies regarding associations between TT and cognition or depression are not consensual with evidences varying from opposite results (for example, Wolf \& Kirschbaum, 2002) and nonlinear associations (Matousek \& Sherwin, 2010), to lack of association (Yaffee et al., 2007). The results herein may provide an explanation for these divergences. However, the mechanisms behind the modulatory effect of cognition on the association between TT and mood status remain to be explored. Also, of note, testosterone occurs both in free and bound forms in blood, with the free-form being considered the "active" form. Higher bioavailable and free testosterone concentrations have been related with better cognitive performance (Yeap et al., 2008). Here we have measured for total testosterone; however, in future studies, it should be explored if levels are mediated by changes in the levels of the binding protein, which would allow 

testosterone and the considered dimensions.

Estradiol distinguished between the weakest cognitive profile (C4) and the remaining groups (C1 to C3); notably, C4 individuals are also the ones that presented the worse well-being and mood (see also Supplementary data, partial correlations). E2 has been associated with increased cognitive performance among older women and men, possibly due to beneficial effects of E2 (treatment) on frontal lobe related tasks (Wolf \& Kirschbaum, 2002). This is also in line with recent studies indicating a modulatory effect of E2 on PFC and amygdala activity in both rats and humans (Zeidan et al., 2011). Furthermore, in animal studies, recent evidence has suggested an antidepressant role of E2 in rats (Yang et al., 2014). This hormone has important effects on brain chemistry and functions fundamental for cognitive processing; however, results are not necessarily straightforward. For example, Yaffee, Lui, Zmuda, and Cauley (2002) reported that lower levels of E2 are related with a decrease in global cognitive function in both older males and females. However, subsequent work indicated that, while total estradiol levels were associated with worse cognitive scores on some executive level tests, bioavailable estradiol levels were not associated with cognitive function (Yaffee et al., 2007). Overall, studies warrant continued research, possibly also taking into account not only distinct cognitive dimensions, but also other aspects, with impact on cognitive performance, such as mood (Santos et al., 2014), and hormone bioavailability.

The hormones FSH and LH appeared to be the ones with the least impact on the domains assessed, but some considerations are of note, particularly regarding the partial correlations' findings. When controlling for age and education, a trend for a significant negative association was observed between overall sleep quality and LH serum levels. These findings are consistent with the in perimenopausal women (Murphy \& Campbell, 2007), and a plausible association between the action of gonadotropin hormones and chronobiological rhythms (Sowers et al., 2008). This may be due to the presence of LH receptors in the hypothalamus and in the pineal gland, known to be involved in the production of melatonin, which in turns regulates the sleep-wake-cycle (Murphy \& Campbell, 2007). In males, FSH and LH serum levels were associated with a poorer executive functioning, in models adjusted for age and education. While few associations have been reported between FSH levels and cognition, there is higher consensus regarding the association between LH and cognition, with research mainly focusing on neurodegenerative diseases and cognitive impairment (Bimonte-Nelson, Acosta, \& Talboom, 2010; Fuller, Tan, \& Martins, 2007; Hyde et al., 2010). Based on our findings, it can be postulated that the physiological significance of gonadotropins goes beyond their reproductive function and that these may be biological markers of interest in age-related cognitive processes: however, further studies with larger cohorts are necessary and warranted.

It is particularly intriguing that the most significant findings were noted in males, with most effects observed for executive function. It has been shown that sex hormones affect multiple aspects of central neuronal function. The synthesis and clearance of neurotransmitters have repercussions in the sympathetic/parasympathetic tone and in hypothalamic-pituitary-adrenocortical (HPA) axis function, both of which are known to influence cognition (Duncko, Johnson, Merikangas, \& Grillon, 2009; Feldman et al., 2002). Certainly more research is needed to understand the neurobiological mechanisms underlying the more pronounced associations between hormone levels and measures of cognition, depression and well-being in men compared

The current study presents strengths including a populationbased sampling, thorough hormone profile (other than the to more finely dissect the relationship between free- and boundreported relationship between LH levels and poorer sleep quality to women. traditionally studied testosterone and estradiol), application of a battery of neurocognitive/psychological tests, and being conducted in a much less educated sample than typically reported. Nonetheless, some study limitations and further directions should be addressed. As it was a cross-sectional design, it is not possible to establish causal relationships between hormones levels and the studied variables. Another issue relates to the sample size. Having this in consideration, the analysis was conducted with the concern of maximizing the statistical power; thus, dimensions were created with the purpose of reducing the number of variables, with a minimum loss of information. As such, results should still be interpreted with caution. Further studies with larger sample sizes and in other cohorts may contribute to a more clear understanding of the observed associations. Furthermore, neuroimaging data could also greatly add to the present findings, elucidating on brain structural and functional correlates. Overall, the findings herein reported strengthen the idea that the less explored PRL hormone plays a role sive mood and well-being in aging, warranting further research.

\section{Conflict of interest}

The authors have nothing to disclose.

\section{Funding}

The work was funded by the European Commission (FP7): Q6 497 "SwitchBox" (Contract HEALTH-F2-2010-259772) and co-financed by the Portuguese North Regional Operational Program (ON.2-O Novo Norte) under the National Strategic Reference Framework (QREN), through the European Regional Development Fund (FEDER). NCS is supported by a "SwitchBox" project post-doctoral fellowship, TCC by a Fundação para a Ciência e Tecnologia (FCT, Portugal) doctoral fellowship, PSM and CPN by a "MyHealth" project (Contract DoIT-13853) doctoral and research fellowship, respectively.

Uncited reference

Fonda, Bertrand, O’Donnell, Longcope, and McKinlay (2005).

\section{Acknowledgements}

The authors are thankful to all colleagues that aided in data collection, to the Clinical Pathology Laboratory at Hospital de Braga, and to the study participants.

\section{References}

Aleman, A., De Vries, W. R., Koppeschaar, H. P., Osman-Dualeh, M., Verhaar, H.J., Samson, M. M., et al.(2001). Relationship between circulating levels of sex hormones and insulin-like growth factor-1 and fluid intelligence in older men. Experimenta Aging Research, 27, 283-291.

Alfaro, S., Serra-Prat, M., Palomera, E., Falcón, I., Cadenas, I., Boquet, X., et al. (2008) Hormonal determinants of depression and cognitive function in independently living elders. Endocrinología y Nutrición, 55, 396-401.

Barrett-Connor, E., \& Goodman-Gruen, D. (1999). Cognitive function and endogenous sex hormones in older women. Journal of the American Geriatrics Society, $47,1289-1293$.

Ben-Jonathan, N., \& Hnasko, R. (2001). Dopamine as a prolactin (PRL) inhibitor Endocrine Reviews, 22, 724-763.

Bimonte-Nelson, H. A., Acosta, J. I., \& Talboom, J. S. (2010). Neuroscientists as cartographers: mapping the crossroads of gonadal hormones, memory and age using animal models. Molecules, 15, 6050-6105.

Bole-Feysot, C., Goffin, V., Edery, M., Binart, N., \& Kelly, P. A. (1998). Prolactin (PRL) and its receptor: Actions, signal transduction pathways and phenotypes observed in PRL receptor knockout mice. Endocrine Reviews, 19, 225-268.

Boss, L., Kang, D., Marcus, M., \& Bergstrom, N. (2013). Endogenous sex hormones and cognitive function in older adults: A systematic review. Western Journal of Nursing Research, 20, 1-39. in specific mechanisms underlying cognitive performance, depres- 
Buysse, D. I., Reynolds, C. F., Monk, T. H., Berman, S. R., \& Kupfe, D. J. (1988). The Pittsburgh sleep quality índex: A new instrument for psychiatric practice and research. Psychiatry Research, 28, 193-213.

Costa, P. S., Santos, N. C., Cunha, P., Palha, J. A., \& Sousa, N. (2013). The use of Bayesian latent class cluster models to classify patterns of cognitive performance in healthy ageing. PLOS ONE, 8, e71940.

Davis, S. R., Panjari, M., \& Stanczyk, F. Z. (2011). DHEA replacement for postmenopausal women. The Journal of Clinical Endocrinology \& Metabolism, 96, 1642-1653.

Duncko, R., Johnson, L., Merikangas, K., \& Grillon, C. (2009). Working memory performance after acute exposure to the cold pressor stress in healthy volunteers. Neurobiology of Learning and Memory, 91, 377-381.

Farr, S. A., Banks, W. A., Uezu, K., Gaski, F. S., \& Morley, J. E. (2004). DHEAS improves learning and memory in aged SAMP8 mice but not in diabetic mice. Life Sciences, $75,2775-2785$

Feldman, H. A., Longcope, C., Derby, C. A., Johannes, C. B., Araujo, A. B., Coviello, A. D., et al. (2002). Age trends in the level of serum testosterone and other hormones in middle-aged men: Longitudinal results from the Massachusetts male aging study. The Journal of Clinical Endocrinology and Metabolism, 87, 589-598.

Fonda, S. J., Bertrand, R., O’Donnell, A., Longcope, C., \& McKinlay, J. B. (2005). Age, hormones, and cognitive functioning among middle-aged and elderly men: Cross-sectional evidence from the Massachusetts Male Aging Study. The Journals of Gerontology Series A: Biological Sciences and Medical Sciences, 60, 385-390.

Fuller, S. J., Tan, R. S., \& Martins, R. N. (2007). Androgens in the etiology of Alzheimer's disease in aging men and possible therapeutic interventions. Journal of Alzheimer's Disease, 12, 129-142.

Goldstein, J. M., Holsen, L. M., Handa, R., \& Tobet, S. (2013). Sex differences in HPA and HPG axes dysregulation in major depressive disorder: The role of shared brain circuitry between hormones and mood. In D. W. Pfaff, \& Y. Christen (Eds.), Multiple origins of sex differences in brain: Research and perspectives in endocrine interactions (pp. 139-163). Berlin/Heidelberg: Springer.

Guigoz, Y., Vellas, B., \& Garry, P. J. (1996). Assessing the nutritional status of the elderly: The mini nutritional assessment as part of the geriatric evaluation. Nutrition Reviews, 54, S59-S65.

Harsh, V., Meltzer-Brody, S., Rubinow, D. R., \& Schmidt, P. J. (2009). Reproductive aging, sex steroids, and mood disorders. Harvard Review Of Psychiatry, 17, 87-102.

Henry, J. F., \& Sherwin, B. (2012). Hormones and cognitive functioning during late pregnancy and postpartum: A longitudinal study. Behavioral Neuroscience, 126, 73-85.

Hermann, M., \& Berger, P. (1999). Hormone replacement in the aging male? Experimental Gerontology, 34, 923-933.

Hyde, Z., Flicker, L., Almeida, O. P., McCaul, K. A., Jamrozik, K., Hankey, G. J., et al. (2010). Higher luteinizing hormone is associated with poor memory recall: The health in men study. Journal of Alzheimer's Disease, 19, 943-951.

Janowsky, J. S. (2006). Thinking with your gonads: Testosterone and cognition. Trends in Cognitive Sciences, 10, 77-82.

Lajud, N., Gonzalez-Zapien, R., Roque, A., Tinajero, E., Valdez, J. J., Clap, C., et al. (2013). Prolactin administration during early postnatal life decreases hippocampal and olfactory bulb neurogenesis and results in depressive-like behavior in adulthood. Hormones and Behavior, 64, 781-789.

Lei, Z. M., Rao, C. V., Kornyei, J. L., Licht, P., \& Hiatt, E. S. (1993). Novel expression of human chorionic gonadotropin/luteinizing hormone receptor gene in brain. Endocrinology, 132, 2262-2270.

Lisansky, J., Fava, G., Buckman, M., Kellner, R., Fava, M., Zielezny, M., et al. (1984) Prolactin, amitriptyline, and recovery from depression. Psychopharmacology, 84, 331-335.

Luetters, C., Huang, M. H., Seeman, T., Buckwalter, G., Meyer, P. M., Avis, N. E., et al. (2007). Menopause transition stage and endogenous estradiol and follicle-stimulating hormone levels are not related to cognitive performance: Cross-sectional results from the study of women's health across the nation (SWAN). Journal of Women's Health, 16, 331-344.

Lunn, P., Austin, S., Prentice, A., \& Whitehea, G. (1984). The effect of improved nutrition on plasma prolactin concentrations and postpartum infertility in lactating Gambian women. The American Journal of Clinical Nutrition, 39, 227-235.

Maggio, M., De Vita, F., Fisichella, A., Colizzi, E., Provenzano, S., Lauretani, F., et al. (2014). DHEA and cognitive function in the elderly. The Journal of Steroid Biochemistry and Molecular Biology, http://dx.doi.org/10.1016/j.jsbmb.2014.03.014

Martínez-Jabaloyas, J. M., Queipo-Zaragozá, A., Ferrandis-Cortes, C., QueipoZaragozá, J. A., Gil-Salom, M., \& Chuan-Nuez, P. (2011). Relationships between sex hormone levels in men over 50 years of age and body composition, bone quality, and quality of life. Actas Urológicas Españolas, 35, 515-522.

Matousek, R. H., \& Sherwin, B. B. (2010). Sex steroid hormones and cognitive functioning in healthy, older men. Hormones and Behavior, 57, 352-359.

Moffat, S. D., Zonderman, A. B., Metter, E. J., Blackman, M. R., Harman, S. M., \& Resnick, S. M. (2002). Longitudinal assessment of serum free testosterone concentration predicts memory performance and cognitive status in elderly men. The Journal of Clinical Endocrinology and Metabolism, 87, 5001-5007.

Morsink, L. F., Vogelzangs, N., Nicklas, B. J., Beekman, A. T., Satterfield, S., Rubin, S. M., et al. (2007). Associations between sex steroid hormone levels and depressive symptoms in elderly men and women: Results from the Health $A B C$ study. Psychoneuroendocrinology, 32, 874-883.

Murphy, P. J., \& Campbell, S. S. (2007). Sex hormones, sleep, and core body temperature in older postmenopausal women. Sleep, 30, 1788-1794.

OECD. (2012). Country statistical profile: Portugal (Organisation for Economic Co-operation and Development iLibrary Web site). http://dx.doi.org/10.1787/csp-prt-Table-2011-1-en

Paulo, A. C., Sampaio, A., Santos, N. C., Costa, P. S., Cunha, P., Zihl, J., et al. (2011) Patterns of cognitive performance in healthy ageing in northern Portugal: A cross-sectional analysis. PLOS ONE, 6, 1-9.

Prull, M. W., Gabrieli, J. D., \& Bunge, S. A. (2000). Age-related changes in memory: A cognitive neuroscience perspective. In F. I. M. Craik, \& T. A. Salthouse (Eds.), The handbook of aging and cognition (pp. 91-153). New York: Psychological Press.

Rathna, K. U., \& Padma, K. (2013). Dehydroepiandrosterone levels and cognitive function in aging. International Journal of Medical Research E' Health Sciences, 2, 101-106.

Ravaglia, G., Forti, P., Maiol, F., Boschi, F., Bernardi, M., Pratelli, L., et al. (1996). The relationship of dehydroepiandrosterone sulfate (DHEAS) to endocrinemetabolic parameters and functional status in the oldest-old. Results from an Italian study on healthy free-living over-ninety-year-olds. The Journal of Clinical Endocrinology and Metabolism, 81, 1173-1178.

Rodrigues, M. A., Verdile, G., Foster, J. K., Hogervorst, E., Joesbury, K., Dhaliwal, S., et al.(2008). Gonadotropins and cognition in older women. Journal of Alzheimer's Disease, 13, 267-274.

Santos, N. C., Costa, P. S., Cunha, P., Cotter, J., Sampaio, A., Zihl, J., et al (2013). Mood is a key determinant of cognitive performance in community-dwelling older adults: A cross-sectional analysis. Age (Dordr), 35, 1983-1993.

Santos, N. C., Costa, P. S., Cunha, P., Portugal-Nunes, C., Amorim, L., Cotter, J., et al (2014). Clinical, physical and lifestyle variables and relationship with cognition and mood in aging: A cross-sectional analysis of distinct educational groups. Frontiers in Aging Neuroscience, 6, 21

Sowers, M. F., Zheng, H., Kravitz, H. M., Matthews, K., Bromberger, J. T., Gold, E. B. et al. (2008). Sex steroid hormone profiles are related to sleep measures from polysomnography and the Pittsburgh Sleep Quality Index. Sleep, 31, 1339-1349.

Torner, L., Tinajero, E., Lajud, N., Quintanar-Stephano, A., \& Olvera-Cortes, E. (2013). Hyperprolactinemia impairs object recognition without altering spatial learning in male rats. Behavioural Brain Research, 252, 32-39.

Vaz Serra, A., Canavarro, M. C., Simões, M. R., Pereira, M., Gameiro, S., Quartilho, M. J., et al. (2006). Estudos psicométricos do instrumento de avaliação da qualidade de vida da organização mundial de saúde (WHOQOL-100) para português de Portugal. Psiquiatria Clinica, 27, 31-40.

Walker, T. L., Vukovic, J., Koudijs, M. M., Blackmore, D. G., Mackay, E. W., Sykes, A. M. et al. (2012). Prolactin stimulates precursor cells in the adult mouse hippocampus. PLOS ONE, 7, e44371.

Wang, Y., Chan, G. L. Y., Holden, J. E., Dobko, T., Mak, E., Schulzer, M., et al. (1998). Age-dependent decline of dopamine D1 receptors in human brain: A PET study. Synapse, 30, 56-61.

Webber, K. M., Perry, G., Smith, M. A., \& Casadesus, G. (2007). The contribution of luteinizing hormone to Alzheimer disease pathogenesis. Journal of Clinical Medicine Research, 5, 177-183.

Wolf, O. T., \& Kirschbaum, C. (1999). Actions of dehydroepiandrosterone and its sulfate in the central nervous system: Effects on cognition and emotion in animals and humans. Brain Research, 30, 264-288.

Wolf, O. T., \& Kirschbaum, C. (2002). Endogenous estradiol and testosterone levels are associated with cognitive performance in older women and men. Hormones and Behavior, 41, 259-266.

Wong, S. Y. S., Leung, J. C., Kwok, T., Ohlsson, C., Vandenput, L., Leung, P. C., et al. (2011). Low DHEAS levels are associated with depressive symptoms in elderly Chinese men: Results from a large study. Asian Journal of Andrology, 13, 898-902.

Yaffee, K. Barnes, D., Lindquist, K. Cauley, J., Simonsick, E. M., Penninx, B., et al. (2007). Endogenous sex hormone levels and risk of cognitive decline in an older biracial cohort. Neurobiology of Aging, 28, 171-178.

Yaffee, K., Lui, L. Y., Zmuda, J., \& Cauley, J. (2002). Sex hormones and cognitive function in older men. Journal of the American Geriatrics Society, 50, 707-712.

Yang, F., Tao, J., Xu, L., Zhao, N., Chen, J., Chen, W., et al. (2014). Estradiol decreases rat depressive behavior by estrogen receptor beta but not alpha: No correlation with plasma corticosterone. Neuroreport, 25, 100-104.

Yang, Y. K., Chiu, N. T., Chen, C. C., Chen, M., Yeh, T. L., \& Lee, I. H. (2003). Correlation between fine motor activity and striatal dopamine D2 receptor density in patients with schizophrenia and healthy controls. Psychiatry Research Neuroimaging, 123, 191-197.

Yeap, B. B., Almeida, O. P., Hyde, Z., Chubb, S. A., Hankey, G. J., Jamrozik, K., et al. (2008). Higher serum free testosterone is associated with better cognitive function in older men, while total testosterone is not. The Health in Men Study. Clinical Endocrinology, 68, 404-412.

Zeidan, M., Igoe, S., Linnman, C., Vitalo, A., Levine, J., Klibanski, A., et al. (2011). Estradiol modulates medial prefrontal cortex and amygdala activity during fear extinction in women and female rats. Biological Psychiatry, 70, 920-927.

Zjacic-Rotkvic, V., Kavur, L., \& Cigrovski-Berkovic, M. (2010). Hormones and aging. Acta Clinica Croatica, 49, 549-554.

66

\section{666
667}

67 \\ 668
669
670}

(1)

\section{(1)}

677

\section{7}

\section{8}

68

\section{8}

68

\section{8}

68

689
690
691
692
693 